Co-located with Laser Science XXVII, the annual meeting of the American Physical Society Division of Laser Science, this year's Frontiers in Optics featured nearly
900 presentations over five days, gathering around 1,700 attendees. The next Frontiers in Optics will be held in Orland, Florida, USA, on 6-10 October 2013.
Rachel Won is at Nature Photonics, Chiyoda Building, 237 Ichigayatamachi, Shinjuku-ku, Tokyo 162-0843, Japan.

$\square$ email:r.won@natureasia.com

\title{
OPTOFLUIDICS
}

\section{On-chip bioassay}

Bioassays are biological experiments that are important for medicine, pharmaceutics and environmental health science, such as in the development of new drugs and the monitoring of pollutants. Pretreatments such as sampling, separation and extraction are needed before a sample can be tested by microorganisms, thus giving on-chip bioassay systems the potential to reduce reagent consumption and detection times.

Recent work by Jae-Sung Kwon and co-workers from Purdue University and Oak Ridge National Laboratory in the USA provides a biocompatible, cost-effective and efficient technique for realizing an on-chip bioassay system (Lab Chip 12, 4955-4959; 2012). The researchers demonstrated that their previously developed technique, known as rapid electrokinetic patterning, which combines a variety of electrokinetic mechanisms such as fluid manipulation, rapid particle assembly and selective particle capture on a chip, can be used to perform speedy and precise aggregation, patterning, translation, trapping and sizebased separation of microorganisms, thus making it attractive for high-performance bioassay systems.

The chip consists of microfluidic chambers sandwiched between a pair of transparent parallel-plate indium tin oxide electrodes. The researchers focused the 1,064-nm-wavelength beam from an $\mathrm{Nd}: \mathrm{YVO}_{4}$ laser on the bottom electrode through an objective, and introduced liquid through the ports on the top electrode. They tested three samples: Shewanella oneidensis (S. oneidensis) MR-1, a 1- $\mu$ m-diameter rod-shaped bacterium, Saccharomyces cerevisiae (S. cerevisiae), a 5- $\mu$ m-diameter spherical fungus, and Staphylococcus aureus (S. aureus), a 1- $\mu$ m-diameter spherical bacterium, and labelled all three with SYTO9 green fluorescent dye.

When the researchers applied a uniform a.c. electric field $(18.69 \mathrm{kHz}, 17.8 \mathrm{~V})$ to the suspension containing MR-1 bacteria, electrohydrodynamic flow caused the bacteria to move towards one of the electrode surfaces. When illuminating

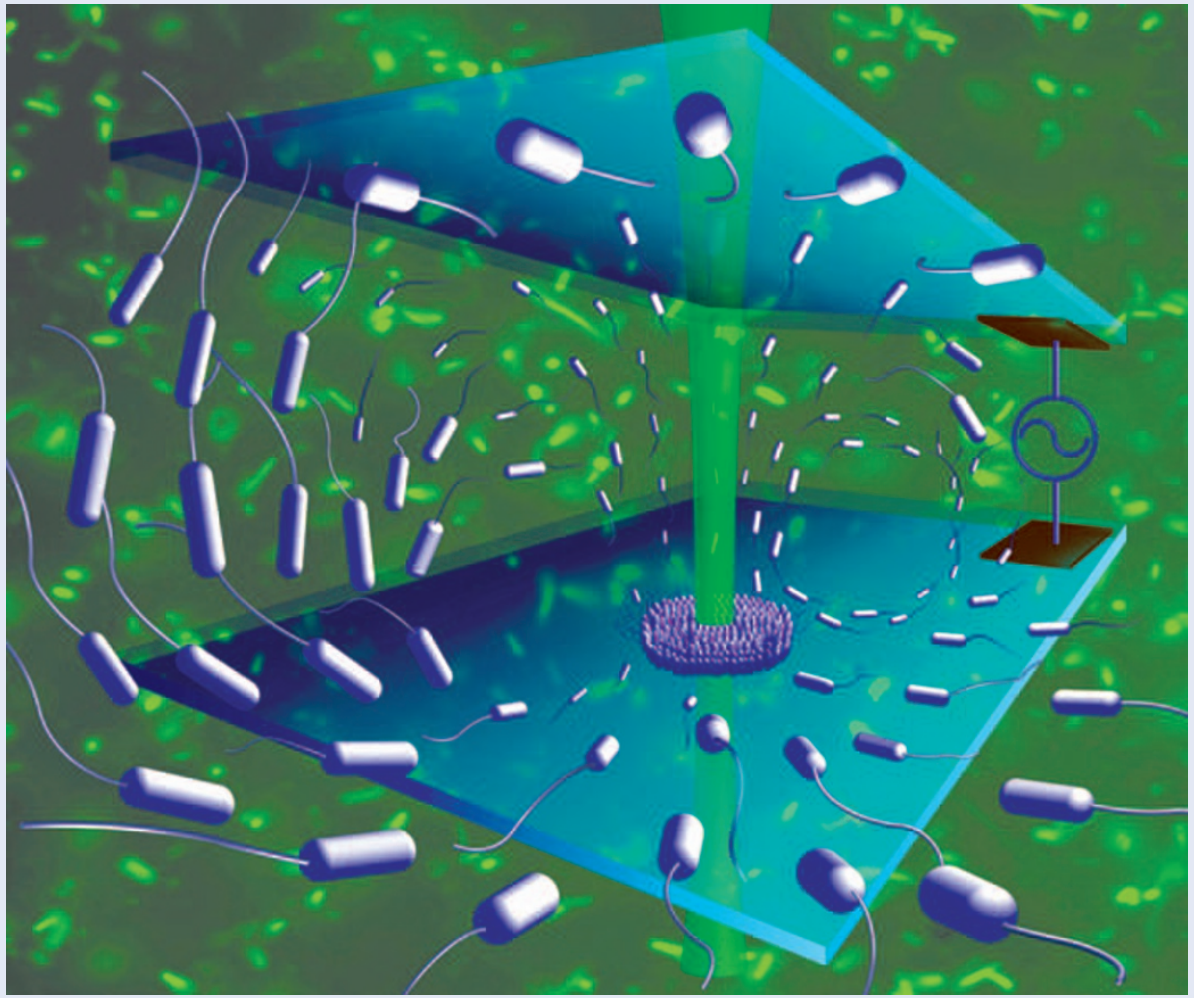

the bottom electrode with a $20 \mathrm{~mW}$ laser, they observed an induced electrothermal vortex due to the generation of temperaturedependent gradients of permittivity and conductivity in the medium, which interact with the applied electric field. The induced toroidal microvortex transported the MR-1 bacteria towards the illumination location on the electrode. As a result, the number of MR-1 bacteria exponentially increased with time in the illuminated area. When the researchers focused two laser spots on the electrode surface, the MR-1 bacteria aggregated at each of the illuminated areas. They also showed that the translation of MR-1 bacteria can be achieved by moving a focused laser across an electrode surface.

The stability of this technique is dependent on a critical frequency that is characterized as the inverse of particle diameter squared for the special case of constant surface charges.
It sets the maximum a.c. frequency at which a stable rapid electrokinetic patterning cluster can be obtained. By simultaneously applying focused laser light and a uniform a.c. electric field above the critical frequency, the researchers have shown that the concentration, patterning and translation of a microorganism can be guaranteed based on the joint effect of electrohydrodynamic flow induced by the a.c. electric field and the electrothermal vortex induced by the laser heating. The dependence of the critical frequency on the surface charge density also allowed them to demonstrate sizebased separation of S. cerevisiae and S. aureus using the same set-up but at different a.c. electric field frequencies. They envisage that this technique will further improve the performance of on-chip bioassay systems.

NORIAKI HORIUCHI 